\title{
Molecular medicine in a changing world
}

It is the essence of science to change the way we think about the world around us and ourselves. It is no surprise that the rapid evolution of biomedical research during the past 20 years (due in large measure to recombinant DNA technology) has changed fundamentally the way medicine approaches the pathogenesis and treatment of disease. Biochemistry and pathology have given way to a new science based on understanding the structure and function of genes and proteins. The art of medicine and the precision of science have come together to create a new discipline - molecular medicine - that holds great promise for the eventual cure of afflictions that haunt humankind - from cardiovascular disease and cancer to resurgent diseases such as tuberculosis and plague.

Molecular medicine encompasses virtually all areas of contemporary biomedical research including gene therapy; the role of angiogenesis in tumour metastasis, rheumatoid arthritis, and infertility; and imaging techniques, such as magnetic resonance imaging. The rapidly growing field of structural biology, with its promise of rational drug design, and new techniques in microsurgery are also part of the new medicine.

We cannot say what the future of science holds but it is possible to make some reasonable predictions. Neuroscience, in stark contradiction to the once solemn certainty that the brain never changes, has shown that the adult brain is considerably more 'plastic' or flexible than anybody thought. This fundamental observation is good news for people with neurological diseases such as Alzheimer's and Parkinson's diseases.

Studies of signal transduction, the fundamental process by which cells talk to one another as intracellular proteins transmit signals from the surface of the cell to the nucleus inside, have opened an entirely new approach to the development of therapeutic agents that can target discrete steps in the body's elaborate pathways of chemical reactions.

The opportunities are endless. Biomedical science is flourishing. Science has not cured cancer with a single bullet and AIDS remains, in large part, intractable. Chronic illnesses, which still defy easy treatment, dominate the medical scene in the developed nations of the world. But looking back over the history of medicine, good omens are there: the future will build on the past, but it will not be a reflection of it.

It was not so very long ago that some of the best advice a senior physician could give a doctor in training was: "Don't just do something. Stand there." Medicine was the art of diagnosis, prognosis, and hoping, as the late Lewis Thomas said, that things would get better by morning. But gradually, we are becoming better at treating those diseases that do not cure themselves.

Nature Medicine intends to be home to papers that bridge the gap between cutting edge biological research and more clinically oriented human investigation. We intend to include the best science in each of the clinical disciplines. Our authors will represent academic research and pharmaceutical and biotechnology laboratories, as well as those labouring in the international fields of public health and epidemiology. Our hope is to be accessible to biomedical researchers and clinicians alike, with an emphasis on clarity of presentation and articles that explicitly put developments in science into a medical context. We cannot promise to get it right the first time out. You will have to let us know how we are doing.

Nature Medicine also recognizes that science policy is an important element in the professional lives of research scientists and physicians. Will academic medicine fare well in a changing environment driven by limited research budgets from governments worldwide? What will be the consequences to research in a world in which cost reduction has assumed the status of the Holy Grail? Nature Medicine will publish news and commentary about the funding and regulation of research in all nations that support biomedical science. Topics such as government policies on fetal research, in vitro fertilization, regulation of biotechnology, and the funding of research and education in academic and private institutions will all be in our purview.

It is true, of course, that biomedical research, for all its achievements and potential, has experienced dashed expectations in the translation of fundamental science into the treatment of human disease. (An AIDS vaccine is an example.) It is also true that the great scientific institutions of the 20th century have grown to such a size that some reduction in force is now inevitable. That will be painful but not necessarily harmful in the long run. The immediate challenge is to find ways to preserve the best of the academic research enterprise while adapting constructively to the reality of constraint and change. But adaptation and change are what biomedical science is all about.

BARBARA J. CULLITON 